\title{
Clinical results of multidisciplinary therapy including palliative posterior spinal stabilization surgery and postoperative adjuvant therapy for metastatic spinal tumor
}

Hiroshi Uei ${ }^{*}$, Yasuaki Tokuhashi, Masafumi Maseda, Masahiro Nakahashi, Hirokatsu Sawada, Enshi Nakayama and Hirotoki Soma

\begin{abstract}
Background: Surgeries performed for metastatic spinal tumor are mostly palliative and are controversial for patients with short life expectancy. We investigated whether palliative posterior spinal stabilization surgery with postoperative multidisciplinary therapy results in improvement of life prognosis and activities of daily living (ADL) in patients with metastatic spinal tumor.

Methods: The subjects were 55 patients who underwent palliative posterior-only instrumentation surgery for metastatic spinal tumor at our hospital between 2012 and 2015. Postoperative survival, early paralysis improvement, ADL improvement, and rate of discharge to home were examined.
\end{abstract}

Results: The patients included 37 males and 18 females, and the mean age at the time of surgery was 66.8 years old. The mean Tokuhashi score was 7.1, the mean spinal instability neoplastic score (SINS) was 9.4, and the epidural spinal cord compression scale (ESCCS) was grade 3 in 20 patients (36.3\%). The mean Barthel index for ADL was 48.7. The median postoperative survival time determined using the Kaplan-Meier method was 12.0 months (95\% confidence interval 2.4-21.5). Regarding improvement of paralysis, the modified Frankel scale was improved by one grade or more or grade E was maintained in 35 patients (63.6\%), whereas paralysis aggravated in 2 (3.6\%). In surgery, conventional posterior decompression and fixation were applied in 31 patients (56.3\%), and minimally invasive spine stabilization was applied in 24 (43.6\%). Postoperative chemotherapy was performed in 31 patients (56.3\%), radiotherapy was used in 38 (69.0\%), and a bone-modifying agent was administered in 39 (70.2\%). Regarding ADL, the mean Barthel index improved from 48.5 before surgery to 74.5 after surgery. Thirty-seven patients (67.2\%) were discharged to home.

Conclusions: ADL improved and allowed discharge to home, and postoperative adjuvant therapy could be administered at a high rate in patients who received palliative posterior spinal stabilization surgery. Survival time extended beyond the preoperative life expectancy in many patients. Patients with a metastatic spinal tumor have short life expectancy and paralysis caused by spinal instability and spinal cord compression. However, multidisciplinary therapy including palliative posterior spinal stabilization surgery with reduced invasiveness and postoperative adjuvant therapy are effective in these patients.

Keywords: Spinal metastases, Palliative surgery, Tokuhashi score, Minimally invasive spine stabilization, Multidisciplinary therapy

\footnotetext{
* Correspondence: uei.hiroshi@nihon-u.ac.jp

Department of Orthopaedic Surgery, Nihon University School of Medicine,

30-1 Oyaguchi Kami-cho, Itabashi-ku, Tokyo 173-8610, Japan
} 


\section{Background}

Most cases of metastatic spinal tumor are systemic conditions with few treatment options [1-3]. Cases of symptomatic spinal metastasis have increased because the survival time of patients with metastatic cancer has been extended by advances in cancer therapy [4]. Metastatic spinal tumor destroys the spine, which induces collapse of spinal support, infiltration into the spinal cord and cauda equina, and compression, which cause pain, paralysis, and disruption of activities of daily living (ADL). Surgeries performed for metastatic spinal tumor are mostly palliative, and use of these procedures in patients with short life expectancy is controversial [5].

Surgical treatment for metastatic spinal tumor with reduced invasiveness is now available, including minimally invasive spine stabilization (MISt) with percutaneous pedicle screws (PPSs) [6-8] and balloon kyphoplasty [9]. Cancer chemotherapy has also advanced [10], and bonemodifying agents [11] for bone metastasis have been introduced. However, only a few studies have investigated whether these new treatments for metastatic spinal tumor contribute to life expectancy and ADL improvement. In this study, we examined use of not only conventional posterior decompression and fixation surgery but also MISt as palliative surgery and the frequency of postoperative adjuvant therapy for metastatic spinal tumor, with a focus on how much MISt contributed to decreasing surgical stress, early paralysis improvement, extended survival, ADL improvement, and rate of discharge to home.

\section{Methods}

\section{Patient population}

After institutional review board approval was obtained, we reviewed our institutional database for patients who had undergone surgery for metastatic spinal tumors. The subjects were 55 patients who underwent palliative surgery for metastatic spinal tumor at our hospital between 2012 and 2015. Inclusion criteria were patients planned for palliative surgery for metastatic spinal tumors that were treated with posterior-only instrumentation. Patients were excluded if they had concurrent anterior fusion surgery. Demographic data, presenting symptoms, and radiographic studies were reviewed.

\section{Surgical indication}

The indications for palliative surgery for metastatic spinal tumor $[1,2]$ are (1) intractable pain due to spinal instability or threat of instability defined by SINS [12], (2) spinal paralysis such as any change in the motor examination, and (3) radiation-resistant cancer such as kidney cancer or thyroid cancer. The exclusion criteria are (1) case indicated for total en bloc spondylectomy, (2) life expectancy $<6$ months and responsive to narcotic analgesics or markedly responsive to radiotherapy, and (3) poor general condition (Karnofsky performance status $\geq 3$ ) and reduced will to live. Patients were treated primarily with MISt as much treatment as possible was provided (Figs. 1 and 2). The exclusion criteria for not treatable with MISt were (1) lesions in the occipital over the cervical region and (2) difficulty confirming the pedicle of the vertebral arch under a fluoroscope or in PPS insertion. When impossible to treat with MISt, patients were treated with conventional posterior decompression and fixation surgery.

\section{Outcome evaluation}

The evaluation items were (1) postoperative survival time; (2) paralysis improvement; (3) surgical procedure, including number of levels fused, operative time, intraoperative blood loss, and complications; (4) use of postoperative adjuvant therapy with chemotherapy, radiotherapy, and bone-modifying agents; (5) change in Barthel index for ADL from before surgery to the highest value after surgery; (6) rate of discharge to home; and (7) VAS at 2 weeks after surgery. With respect to postoperative adjuvant therapy, we tried to perform radiotherapy after surgery excluding radiation-resistant cancer, as long as it is not inconvenient for treatment of the primary cancer. The criterion for discharge to home was Barthel index $\geq 70$ or availability of sufficient care by family members for cases with Barthel index $<70$. Statistical analysis was performed using SPSS v.19.0 (SPSS Inc., Chicago, IL, USA), with the significance level at $5 \%$.

\section{Results}

The patients included 37 males (67.2\%) and 18 females (32.7\%), and the mean age at the time of surgery was 66.8 years old (Table 1 ). The primary lesion was lung cancer in 11 patients (20.0\%), liver cancer in 9 (16.3\%), prostate cancer in 6 (10.9\%), myeloma in 5 (9.0\%), kidney in $4(7.2 \%)$, thyroid in $4(7.2 \%)$, lymphoma in 3 (5.4\%), gallbladder in $3(5.4 \%)$, breast in $2(3.6 \%)$, sarcoma in $2(3.6 \%)$, others in $5(9.0 \%)$, and unknown in $1(1.8 \%)$. The level of the main lesion was the cervical spine in 9 patients (16.3\%), thoracic spine in 34 (58.1\%), and lumbar spine in $14(25.4 \%)$. The grade of preoperative paralysis on the modified Frankel scale (Table 2) [13] was A in 2 patients (3.6\%), B in 1 (1.8\%), C in 19 (34.5\%), D1 in 15 (27.2\%), D2 in $1(1.8 \%), \mathrm{D} 3$ in 10 (18.1\%), and $\mathrm{E}$ in 7 (12.7\%). The mean preoperative visual analogue scale (VAS) for pain was 4.6. The Tokuhashi score [1-3] was $0-8$, indicating life expectancy < 6 months, in 40 patients $(72.7 \%) ; 9-11$, life expectancy $\geq 6$ months, in $9(16.3 \%)$; and $12-15$, life expectancy $\geq 1$ year, in 6 (10.9\%); with an overall mean of 7.1. The spinal instability neoplastic score (SINS) [12] was $0-6$, indicating instability, in 6 patients $(10.9 \%)$; 

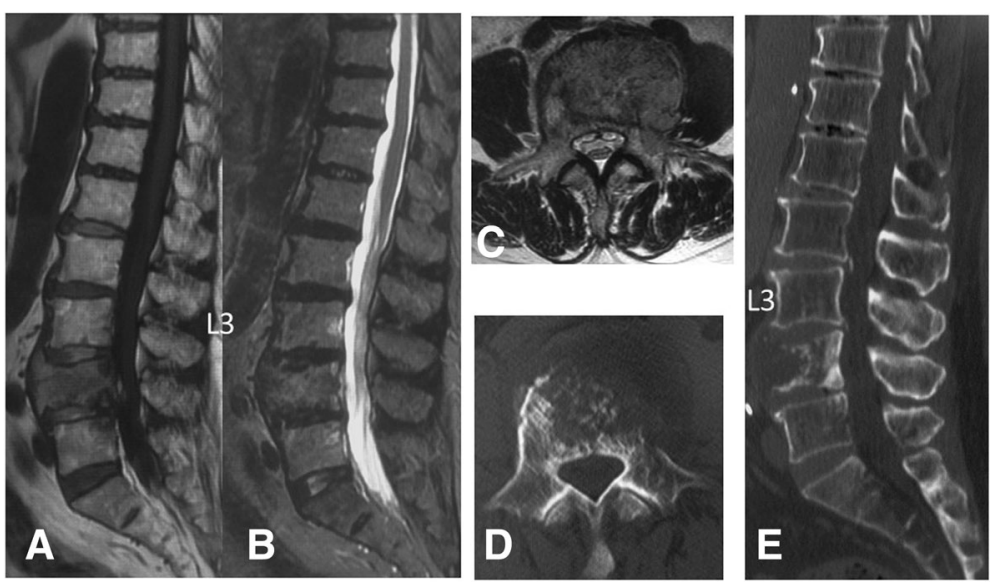

Fig. 1 A 64-year-old man with metastasis of liver cancer to the 4th and 5th lumbar vertebrae. The Tokuhashi score was 3. Minimally invasive spine stabilization (MISt) without decompression (L2-S2Al) was applied. The operative time was $182 \mathrm{~min}$, and blood loss was $152 \mathrm{ml}$. The grade of paralysis improved from D1 before surgery to E after surgery. a Sagittal view on preoperative T1-weighted MRI. b Sagittal view on preoperative STIR MRI. c Axial view at $L 4$ on preoperative T2-weighted MRI. $\mathbf{d}$ Axial view at $L 4$ on preoperative plain $C$. e Sagittal view on preoperative plain $\subset T$

7-12, threat of instability, in 48 (87.2\%); and 13-18, stability, in one (1.8\%); with a mean of 9.4. The epidural spinal cord compression scale (ESCCS) [14] for nerve compression was grade 0 , indicating a tumor restricted to the bone, in one patient (1.8\%); grade 1a, infiltration in the spinal canal, in 4. (7.2\%); grade $1 \mathrm{~b}$ in $4(7.2 \%)$; grade $1 \mathrm{c}$ in 3 (5.4\%); grade 2, nerve compression, in 23 (41.8\%); and grade 3, marked exclusion of the nerve, in 20 (36.3\%). The mean preoperative Barthel index [15] for ADL was 48.7.

The median postoperative survival time determined using the Kaplan-Meier method was 12.0 months (95\% confidence interval 2.4-21.5) (Fig. 3). As for two sarcoma patients, they died 10 and 15 months after surgery. Regarding five myeloma patients, they were all alive at 4 to 30 months (mean 11.6 months) after surgery.

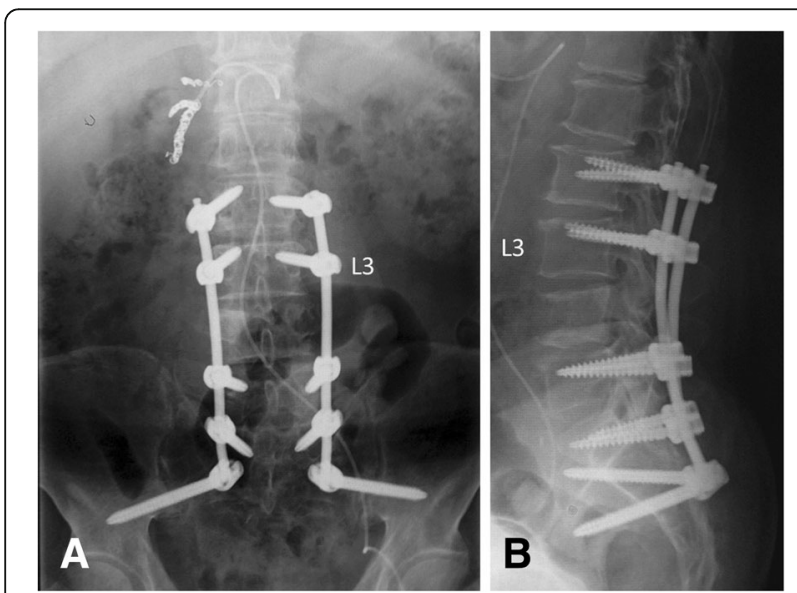

Fig. 2 Postoperative day 3. a Posteroanterior view on postoperative radiography. b Lateral view on postoperative radiography
Paralysis was improved by $\geq 1$ grade or grade $\mathrm{E}$ was maintained in 35 patients (63.6\%) (Table 3). The grade was $C$ or lower before surgery in 22 patients (40.0\%), but the number of patients with these grades decreased to $12(21.8 \%)$ after surgery. The postoperative grade was $\mathrm{E}$ in 21 patients (38.1\%). Paralysis aggravated in 2 patients (3.6\%).

The surgical procedure was conventional posterior decompression and fixation in 31 patients (56.3\%) and MISt in 24 (43.6\%) (Table 4). The mean operative time was $201 \mathrm{~min}$, the mean intraoperative blood loss was $474 \mathrm{ml}$, and the mean number of levels fused was 5.5. Perioperative complications included massive bleeding of $\geq 1500 \mathrm{ml}$ during surgery in 5 patients (9.0\%), death within 30 days after surgery in 3 patients (5.4\%), epidural hematoma after surgery in $2(3.6 \%)$, and wound dehiscence in 2 (3.6\%). With regard to two postoperative epidural hematoma patients, they were treated with surgical evacuation immediately after diagnosis. As for two patients with wound dehiscence, they were treated with resuture and healed of wound properly.

As postoperative adjuvant therapy, chemotherapy was performed in 31 patients (56.3\%), radiotherapy was used in 38 (69.0\%), and a bone-modifying agent was administered in $39(70.2 \%)$ (Table 5). The mean preoperative VAS for pain improved from 4.6 before surgery to 0.9 after surgery $(P<0.001)$, and the mean preoperative Barthel index for ADL improved from 48.5 before surgery to 74.5 after surgery $(P<0.001)$. In patients with mean preoperative Tokuhashi scores of $\leq 8,9-11$, and $\geq$ 12, the mean Barthel index improved from 47.5 to 65.9 $(P<0.001), 52.5$ to $98.5(P=0.01)$, and 48.0 to $92.0(P<$ 0.001 ), respectively (Fig. 4). Thirty-seven patients (67.2\%) were discharged to home, including $48.8 \%$ (20/ 
Table 1 Baseline characteristics

\begin{tabular}{|c|c|}
\hline Characteristic & Value \\
\hline Patients, $n$ & 55 \\
\hline Age at surgery, mean (range), years & $66.8(26-92)$ \\
\hline \multicolumn{2}{|l|}{ Sex, $n(\%)$} \\
\hline Male & $37(67.2)$ \\
\hline Female & $18(32.7)$ \\
\hline \multicolumn{2}{|l|}{ Metastatic tumor diagnosis, $n(\%)$} \\
\hline Lung & $11(20.0)$ \\
\hline Liver & $9(16.3)$ \\
\hline Prostate & $6(10.9)$ \\
\hline Myeloma & $5(9.0)$ \\
\hline Kidney & $4(7.2)$ \\
\hline Thyroid & $4(7.2)$ \\
\hline Lymphoma & $3(5.4)$ \\
\hline Gallbladder & $3(5.4)$ \\
\hline Breast & $2(3.6)$ \\
\hline Sarcoma & $2(3.6)$ \\
\hline Others & $5(9.0)$ \\
\hline Unknown & $1(1.8)$ \\
\hline \multicolumn{2}{|l|}{ Main level of tumors, $n(\%)$} \\
\hline Cervical & $9(16.3)$ \\
\hline Thoracic & $32(58.1)$ \\
\hline Lumbar & $14(25.4)$ \\
\hline \multicolumn{2}{|c|}{ Preoperative modified Frankel category, $n$ (\%) } \\
\hline A & $2(3.6)$ \\
\hline B & $1(1.8)$ \\
\hline C & $19(34.5)$ \\
\hline D1 & $15(27.2)$ \\
\hline D2 & $1(1.8)$ \\
\hline D3 & $10(18.1)$ \\
\hline E & $7(12.7)$ \\
\hline Visual analogue scale (range) & $4.6(1-10)$ \\
\hline \multicolumn{2}{|l|}{ Tokuhashi score, $n(\%)$} \\
\hline $0-8$ & $40(72.7)$ \\
\hline $9-11$ & $9(16.3)$ \\
\hline $12-15$ & $6(10.9)$ \\
\hline \multicolumn{2}{|l|}{ Spinal instability neoplastic score, $n(\%)$} \\
\hline $0-6$ & $6(10.9)$ \\
\hline $7-12$ & $48(87.2)$ \\
\hline $13-18$ & $1(1.8)$ \\
\hline \multicolumn{2}{|c|}{ Epidural spinal cord compression scale, $n(\%)$} \\
\hline 0 & $1(1.8)$ \\
\hline $1 \mathrm{a}$ & $4(7.2)$ \\
\hline $1 b$ & $4(7.2)$ \\
\hline $1 c$ & $3(5.4)$ \\
\hline
\end{tabular}

Table 1 Baseline characteristics (Continued)

\begin{tabular}{ll}
\hline Characteristic & Value \\
\hline 2 & $23(41.8)$ \\
3 & $20(36.3)$ \\
Preoperative Barthel index, mean (range) & $48.7(0-100)$ \\
\hline
\end{tabular}

41), $66.6 \%$ (6/9), and $100 \%$ of patients with preoperative Tokuhashi scores of $\leq 8,9-11$, and $\geq 12$, respectively.

\section{Discussion}

Health conditions and quality of life (QOL) may be improved in survivors of advanced cancer with sufficient survivorship care [16]. Prevention of spinal metastasis-induced skeletal-related events (SREs) is an important aspect of survivorship care because pathological fracture is associated with increased mortality [17]. Surgery alone is insufficient to strengthen survivorship care for patients with metastatic spinal tumor, and administration of adjuvant therapy including radiotherapy, chemotherapy appropriate for the cancer type, and a bone-modifying agent (bisphosphonate or denosumab) followed by hormone replacement are necessary. However, there has been a tendency to focus only on the surgical method and short-term improvement of paralysis in evaluation of therapeutic effects.

Given that improvement of outcomes by surgical treatment alone is limited, we used chemotherapy, radiotherapy, and bone-modifying agents after surgery in 56, 69, and $70 \%$ of cases in this study, respectively. Of the cases excluding radiation-resistant cancer such as kidney and thyroid cancer, radiotherapy was performed in 38 out of 47 patients (80\%). This resulted in improved ADL and greater discharge to home, compared with findings in our previous study [1]. The rate of conventional posterior decompression and fixation surgery decreased to $56 \%$, whereas MISt increased. Performance of MISt at an early stage may have improved ADL, allowed discharge to home, and facilitated use of postoperative adjuvant therapy.

Table 2 Modified Frankel grading scale

\begin{tabular}{|c|c|}
\hline Grade & Neurological status \\
\hline $\bar{A}$ & Complete motor and sensory loss \\
\hline B & Preserved sensation only, voluntary motor function absent \\
\hline C & $\begin{array}{l}\text { Preserved motor less than fair grade (nonfunctional for any } \\
\text { useful purpose) }\end{array}$ \\
\hline D1 & $\begin{array}{l}\text { Preserved motor at lowest functional grade }(3+/ 5+) \text { and/or } \\
\text { with bowel or bladder dysfunction }\end{array}$ \\
\hline D2 & $\begin{array}{l}\text { Preserved motor at midfunctional grade }(3+\text { to } 4+/ 5+) \text { and/or } \\
\text { neurologic bowel or bladder dysfunction }\end{array}$ \\
\hline D3 & $\begin{array}{l}\text { Preserved motor at high-function grade }(4+\text { to } 5+) \text { and normal } \\
\text { voluntary bowel or bladder function }\end{array}$ \\
\hline$E$ & $\begin{array}{l}\text { Complete motor and sensory function normal (may still have } \\
\text { abnormal reflexes) }\end{array}$ \\
\hline
\end{tabular}




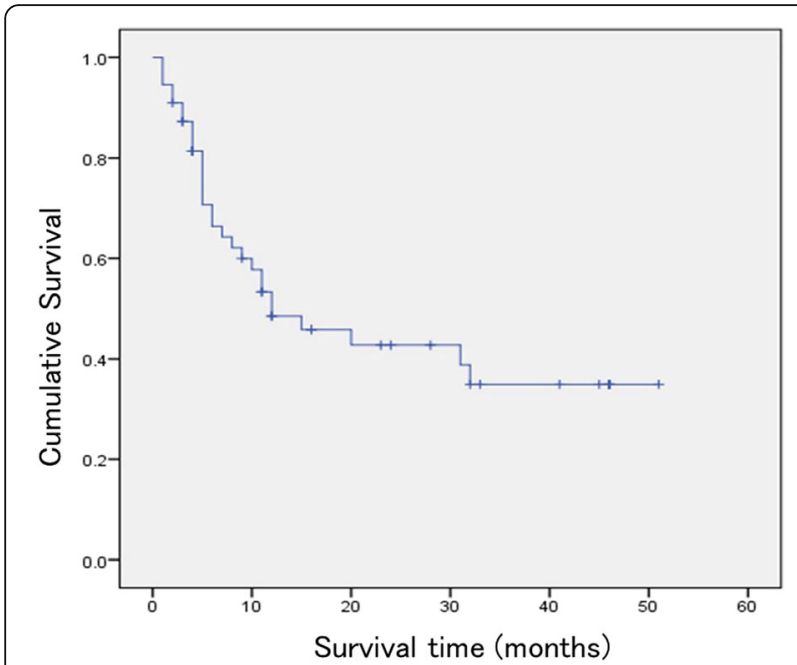

Fig. 3 Kaplan-Meier curve for survival of patients after palliative surgery for metastatic spinal tumor. The median survival time was 12.0 months (95\% confidence interval 2.2-21.8)

Accurate preoperative prediction of the outcome of surgery for metastatic spinal tumor is difficult $[1-3,5]$, and this makes the significance of palliative surgery for patients with short life expectancy controversial. The mean Tokuhashi score was 7.1 in all patients and the score was $0-8$ in 40 patients (72.7\%), indicating life expectancy $<6$ months. Performance of palliative surgery in patients with such a short life expectancy requires consideration of the risk of complications, expected benefits, and medical costs [18]. However, there is also the opinion that palliative surgery should be performed [19] because improvement of ADL by surgery may increase the opportunity for adjuvant therapy after surgery and indirectly extend survival. We also consider that survival can be extended beyond the preoperative life expectancy and ADL can be improved by multidisciplinary therapy including palliative posterior spinal stabilization surgery with reduced invasiveness and postoperative adjuvant therapy. Previous studies of minimally invasive posterior

Table 3 Neurological recovery on the modified Frankel scale

\begin{tabular}{llllllllll}
\hline MFS & $\begin{array}{l}\text { Number } \\
\text { of cases } \\
\text { before } \\
\text { surgery }\end{array}$ & A & B & C & D1 & D2 & D3 & E \\
\cline { 3 - 9 } & 2 & 2 & 0 & 0 & 0 & 0 & 0 & 0 \\
\hline A & 1 & 1 & 0 & 0 & 0 & 0 & 0 & 0 \\
B & 19 & 0 & 1 & 7 & 3 & 1 & 1 & 6 \\
C & 15 & 0 & 0 & 0 & 2 & 4 & 5 & 4 \\
D1 & 1 & 0 & 0 & 0 & 0 & 1 & 0 & 0 \\
D2 & 10 & 0 & 0 & 1 & 0 & 0 & 4 & 5 \\
D3 & 7 & 0 & 0 & 0 & 0 & 0 & 1 & 6 \\
E & 55 & 3 & 1 & 8 & 5 & 6 & 11 & 21 \\
Total & 55 & & & & & & & \\
\hline
\end{tabular}

Table 4 Intraoperative parameters

\begin{tabular}{ll}
\hline Variable & Value \\
\hline Procedures & \\
$\begin{array}{l}\text { Conventional posterior decompression and fixation, } \\
n(\%)\end{array}$ & $31(56.3)$ \\
Minimally invasive spine stabilization, $n(\%)$ & $24(43.6)$ \\
Number of levels fused, mean (range) & $5.5(2-11)$ \\
Operation time, mean (range), (min) & $201(63-371)$ \\
Blood loss, mean (range), (ml) & $474(5-4140)$ \\
Perioperative complications, yes, $n$ (\%) & \\
Massive bleeding (> 1500 ml) & $5(9.0)$ \\
Early death (within 30 days postoperatively) & $3(5.4)$ \\
Epidural hemorrhage & $2(3.6)$ \\
Wound dehiscence & $2(3.6)$ \\
Upper airway obstruction & $1(1.8)$ \\
Acute renal failure & $1(1.8)$ \\
Surgical site infection & $1(1.8)$ \\
Deep vein thrombosis & $1(1.8)$ \\
\hline
\end{tabular}

fixation as palliative surgery have focused on technical aspects, surgical invasiveness, and short-term improvement of paralysis, whereas few have examined outcomes of postoperative survival, ADL, and QOL. Improvement of QOL at 30 days after surgery [6] and median postoperative survival of 11.3 months [7] have been reported. In the current study, the median postoperative survival determined by the Kaplan-Meier method was 12.0 months, and the survival time was longer than the preoperative life expectancy in many patients. The mean Barthel index reflecting ADL improved to 74.5 after surgery, but this was not a marked improvement compared with that in our previous study [1]. In patients with mean preoperative Tokuhashi scores of $\leq 8$, the mean Barthel index improved from 47.5 to 65.9. However, our criterion for discharge to home was Barthel index $\geq 70$ or availability of sufficient care by family members for

Table 5 Outcomes after surgery

\begin{tabular}{ll}
\hline Variable & Value \\
\hline Additional adjuvant therapy, $n$ (\%) & \\
$\quad$ Chemotherapy & $31(56.3)$ \\
Radiotherapy & $38(69.0)$ \\
Bone-modifying agent & $39(70.2)$ \\
Visual analogue scale (range) & $0.9(0-4)$ \\
Postoperative Barthel index, mean (range) & $74.5(0-100)$ \\
Postoperative course, $n$ (\%) & \\
$\quad$ Discharge to home & $37(67.2)$ \\
Transfer to hospice & $11(20.0)$ \\
In-hospital death & $7(12.7)$ \\
\hline
\end{tabular}




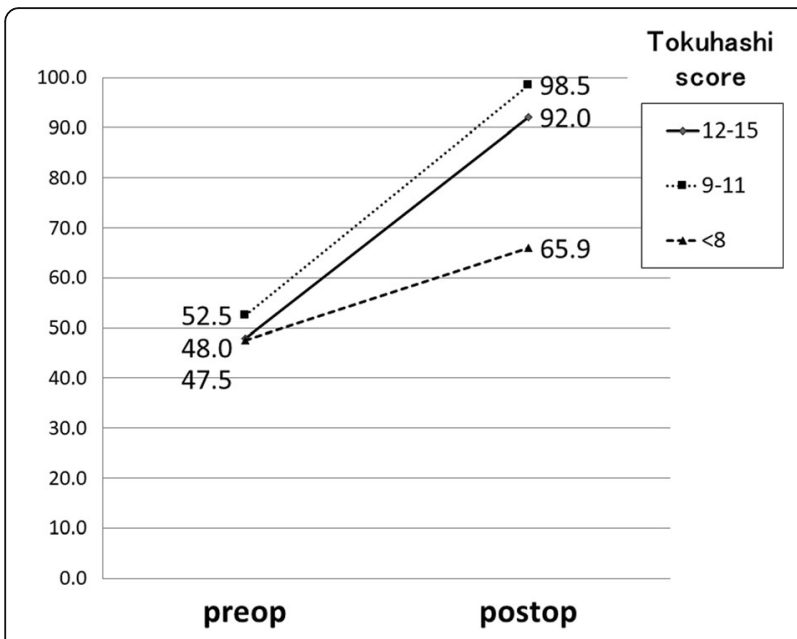

Fig. 4 Changes in Barthel index after surgery in patients classified by Tokuhashi score for life expectancy before surgery. The mean Barthel index in all patients improved from 48.7 before surgery to 74.3 after surgery

cases with Barthel index $<70$.Hence, the rate of discharge to home was $67 \%$, which showed a marked improvement but still not satisfactory.

Chemotherapy, radiotherapy, and treatment with bone-modifying agents can be performed at an outpatient clinic, and this may have contributed to improvement of postoperative patients'QOL. The limitations of this study were that the study design was retrospective without a control group, and there was insufficient evaluation of QOL, but the rate of patients treated with MISt increased, and MISt is advantageous in that the surgical wound is small and the wound heals rapidly. This may have facilitated early postoperative adjuvant therapy and led to the improved rate of discharge to home, which in turn resulted in improved QOL.

\section{Conclusions}

ADL improved and allowed discharge to home, and postoperative adjuvant therapy could be administered at a high rate in patients who received palliative posterior spinal stabilization surgery. Survival time extended beyond the preoperative life expectancy in many patients. Patients with a metastatic spinal tumor have short life expectancy and paralysis caused by spinal instability and spinal cord compression. However, multidisciplinary therapy including palliative posterior spinal stabilization surgery with reduced invasiveness and postoperative adjuvant therapy are effective in these patients.

\section{Abbreviations}

ADL: Activities of daily living; ESCCS: Epidural spinal cord compression scale; MISt: Minimally invasive spine stabilization; QOL: Quality of life; SINS: Spinal instability neoplastic score; SREs: Skeletal-related events

Acknowledgements

Not applicable
Funding

There was no funding for this study.

Availability of data and materials

All data used and analyzed during this study are available from the corresponding author on reasonable request.

\section{Authors' contributions}

$\mathrm{HU}$ performed the study design, analyzed the results, and contributed to the manuscript. YT, MM, MN, HS, EN, and HS contributed to collecting the cases. YT made some meaningful suggestions. All authors reviewed and approved the final submitted version.

\section{Ethics approval and consent to participate}

This study was approved by the Nihon University Institutional Review Board. Written informed consent was obtained from all patients.

Consent for publication

Not applicable

\section{Competing interests}

The authors declare that they have no competing interests.

\section{Publisher's Note}

Springer Nature remains neutral with regard to jurisdictional claims in published maps and institutional affiliations.

Received: 12 December 2017 Accepted: 24 January 2018

Published online: 05 February 2018

\section{References}

1. Tokuhashi Y, Ajiro Y, Umezawa N. Outcome of treatment for spinal metastases using scoring system for preoperative evaluation of prognosis. Spine. 2009;34:69-73. https://doi.org/10.1097/BRS.0b013e3181913f19.

2. Tokuhashi $Y$, Matsuzaki $\mathrm{H}$, Oda $\mathrm{H}$, et al. A revised scoring system for preoperative evaluation of metastatic spine tumor prognosis. Spine. 2005;30: 2186-91.

3. Tokuhashi $Y$, Uei H, Oshima M, et al. Scoring system for prediction of metastatic spine tumor prognosis. World J Orthop. 2014;5:262-71. https:// doi.org/10.5312/wjo.v5.i3.262

4. Rosen LS, Gordon D, Kaminski M, et al. Long-term efficacy and safety of zoledronic acid compared with pamidronate disodium in the treatment of skeletal complications in patients with advanced multiple myeloma or breast carcinoma: a randomized, double-blind, multicenter, comparative trial. Cancer. 2003;98:1735-44. https://doi.org/10.1002/cncr.11701.

5. Verlaan JJ, Choi D, Versteeg A, et al. Characteristics of patients who survived $<3$ months or $>2$ years after surgery for spinal metastases: can we avoid inappropriate patient selection? J Clin Oncol. 2016;34:3054-61. https://doi. org/10.1200/jco.2015.65.1497

6. Miscusi M, Polli FM, Forcato $\mathrm{S}$, et al. Comparison of minimally invasive surgery with standard open surgery for vertebral thoracic metastases causing acute myelopathy in patients with short- or mid-term life expectancy: surgical technique and early clinical results. J Neurosurg Spine. 2015;22:518-25. https://doi.org/10.3171/2014.10.spine131201.

7. Kwan MK, Lee CK, Chan CY. Minimally invasive spinal stabilization using fluoroscopic-guided percutaneous screws as a form of palliative surgery in patients with spinal metastasis. Asian Spine J. 2016;10:99-110. https://doi. org/10.4184/asj.2016.10.1.99.

8. Zairi F, Arikat A, Allaoui M, et al. Minimally invasive decompression and stabilization for the management of thoracolumbar spine metastasis. J Neurosurg Spine. 2012;17:19-23. https://doi.org/10.3171/2012.4.spine111108.

9. Bouza C, Lopez-Cuadrado T, Cediel P, et al. Balloon kyphoplasty in malignant spinal fractures: a systematic review and meta-analysis. BMC Palliat Care. 2009;8:12. https://doi.org/10.1186/1472-684x-8-12.

10. Brahmer J, Reckamp KL, Baas $P$, et al. Nivolumab versus docetaxel in advanced squamous-cell non-small-cell lung cancer. New Engl J Med. 2015; 373:123-35. https://doi.org/10.1056/NEJMoa1504627.

11. Lipton A, Fizazi K, Stopeck AT, et al. Effect of denosumab versus zoledronic acid in preventing skeletal-related events in patients with bone metastases by baseline characteristics. Eur J Cancer. 2016;53:75-83. https://doi.org/10. 1016/j.ejca.2015.09.011. (Oxford, England : 1990) 
12. Fisher CG, DiPaola CP, Ryken TC, et al. A novel classification system for spinal instability in neoplastic disease: an evidence-based approach and expert consensus from the Spine Oncology Study Group. Spine. 2010;35: E1221-9. https://doi.org/10.1097/BRS.0b013e3181e16ae2.

13. Bradford DS, McBride GG. Surgical management of thoracolumbar spine fractures with incomplete neurologic deficits. Clin Orthop Relat Res. 1987; 218:201-16.

14. Bilsky MH, Laufer I, Fourney DR, et al. Reliability analysis of the epidural spinal cord compression scale. J Neurosurg Spine. 2010;13:324-8. https:// doi.org/10.3171/2010.3.spine09459.

15. Mahoney Fl, Barthel DW. Functional evaluation: the Barthel index. Md State Med J. 1965;14:61-5.

16. Thong MS, Mols F, Coebergh JW, et al. The impact of disease progression on perceived health status and quality of life of long-term cancer survivors. J Cancer Surviv. 2009:3:164-73. https://doi.org/10.1007/s11764-009-0094-1.

17. Coleman RE. Clinical features of metastatic bone disease and risk of skeletal morbidity. Clin Cancer Res. 2006;12:6243s-9s. https://doi.org/10.1158/10780432.ccr-06-0931.

18. Turner I, Minhas Z, Kennedy J, et al. Cost of surgery for symptomatic spinal metastases in the United Kingdom. World Neurosurg. 2015;84:1235-43. https://doi.org/10.1016/j.wneu.2015.06.008.

19. Chong S, Shin SH, Yoo H, et al. Single-stage posterior decompression and stabilization for metastasis of the thoracic spine: prognostic factors for functional outcome and patients' survival. Spine J. 2012;12:1083-92. https:// doi.org/10.1016/j.spinee.2012.10.015

\section{Submit your next manuscript to BioMed Central and we will help you at every step:}

- We accept pre-submission inquiries

- Our selector tool helps you to find the most relevant journal

- We provide round the clock customer support

- Convenient online submission

- Thorough peer review

- Inclusion in PubMed and all major indexing services

- Maximum visibility for your research

Submit your manuscript at www.biomedcentral.com/submit

) Biomed Central 